\title{
Ionization of hydrogen and deuterium atoms in thermal energy collisions with metastable $\mathrm{He}^{*}\left(2^{3} \mathrm{~S}\right)$ atoms
}

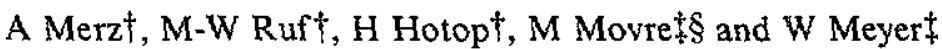 \\ Fachbereich Physik $\dagger$ und Chemieł, Universitāt Kaiserslautern, 67653 Kaiserslautern, \\ Germany
}

Received 19 May 1994

\begin{abstract}
We report experimental and theoretical results for the basic autoionization processes $\mathrm{He}^{*}\left(2^{\prime} \mathrm{S}\right)+\mathrm{H}^{\prime} \mathrm{D}\left(1^{2} \mathrm{~S}\right)$, leading to Penning $\left(\rightarrow \mathrm{He}+\mathrm{H}^{+} / \mathrm{D}^{+}+\mathrm{e}^{-}(\Theta, \varepsilon)\right)$ and associative ionization $\left(\rightarrow \mathrm{HeH}^{+} / \mathrm{HeD}^{+}\left(v^{+}, J^{+}\right)+\mathrm{e}^{-}(\Theta, \varepsilon)\right)$. The experimental electron energy spectra, measured at thermal collision energies $\left(\bar{E}_{\mathrm{rel}} \approx 50 \mathrm{meV}\right)$, show an angular-dependent behaviour which is due to the interplay between the orientation of the collision complex at the moment of autoionization-as referred to the relative velocity direction-and a strongly non-isotropic internal angular distribution for electron emission from the excited quasimolecule. The $a b$ initio work on $\mathrm{He}^{*}\left(2^{3} \mathrm{~S}\right)+\mathrm{H} / \mathrm{D}$ includes electron angular momentum dependent complex (resonance-to-continuum) coupling elements, revealing substantial contributions of autoionization into the electronic $d$ wave. Comparison between the angledifferential experimental electron spectra. measured with an energy resolution of $30 \mathrm{meV}$, and the corresponding theoretical distributions, obtained in the framework of the local complex potential theory, yields good agreement for both isotopes.
\end{abstract}

\section{Introduction}

Studies of collisional autoionization processes represent an active field of research (Siska 1993, Brunetti and Vecchiocattivi 1993, Klyucharev 1993, Bardou et al 1992, Merz et al 1993, Urbain 1993). Associative ionization processes in collisions between excited alkali atoms at low and ultralow energies have been a topic of special recent interest (Weiner et al 1990, Meijer et al 1991, Dulieu et al 1991, Lett et al 1991, Julienne et al 1993, Urbain 1993), partly due to their one-electron character, but also because of the availability of suitable excitation lasers and laser-cooling schemes. The theoretical interpretation of the bi-excited alkali systems is, however, quite complicated (Dulieu et al 1991), and a large number of potential curves are in principle involved (Magnier et al 1993). In contrast, the present study addresses one of the simplest autoionizing collision systems, leading to both Penning (PI) and associative ionization (AI):

$$
\mathrm{He}^{*}\left(2^{3} \mathrm{~S}_{1}\right)+\mathrm{H} / \mathrm{D}\left(1^{2} \mathrm{~S}_{1 / 2}\right)-\left[\begin{array}{l}
\mathrm{He}+\mathrm{H}^{+} / \mathrm{D}^{+}+\mathrm{e}^{-}(\Theta, \varepsilon) \\
-\mathrm{HeH}^{+} / \mathrm{HeD}^{+}\left(v^{+}, J^{+}\right)+\mathrm{e}^{-}(\Theta, \varepsilon)
\end{array}\right.
$$

Ionization occurs from a single entrance channel potential $V^{*}(R)$ with ${ }^{2} \Sigma^{+}$symmetry into an electronic continuum built on a single exit channel potential $V^{+}(R)$ with ${ }^{1} \Sigma^{+}$

§ Permanent address: Institute of Physics, University of Zagreb, 41000 Zagreb, Croatia. 
symmetry. In this paper, we extend our earlier experimental (Merz et al 1992, 1993) and theoretical work (Merz et al 1993, Movre and Meyer 1994) on $\mathrm{He}^{*}\left(2^{3} \mathrm{~S}_{1}\right)+\mathrm{H}$ to collisions with the heavier isotope deuterium. By comparing angle-differential high resolution electron energy spectra with the results of quantum mechanical $a b$ initio calculations, we get deeper insight into the dynamics of electron emission from excited quasi-molecules $\left(\mathrm{HeH}^{*}, \mathrm{HeD}^{*}\right)$. Although semiclassical and wKB studies are useful in the interpretation of experimental results (Miller 1970, Gerber and Niehaus 1976, Bieniek et al 1990), recent examples have demonstrated (Merz et al 1990, 1993, Movre and Meyer 1994) that a proper quantal treatment of electronic coupling as a function of angular momenta is required to obtain full agreement with the experimental electron spectra. The close accord between our theoretical and experimental results verifies that Penning ionization processes for systems with distinctly separated entrance and exit channel potential curves are well described by the theory based on the Born-Oppenheimer and the local complex potential approximations, as formulated more than twenty years ago (Nakamura 1968, 1969, Miller 1970).

The paper is organized as follows: we begin with a rather detailed description of our experimental set-up and the evaluation procedure leading from the raw experimental data to electron spectra which can be compared with theory. Starting with the results of Waibel et al (1988), we summarize the present situation for the benchmark system $\mathrm{He}^{*}\left(2^{3} \mathrm{~S}_{1}\right)+\mathrm{H}\left(1^{2} \mathrm{~S}_{1 / 2}\right)$ and then discuss the new experimental and theoretical work on deuterium.

\section{Experimental set-up}

Our experimental set-up, shown in figure 1, involves crossed atomic beams and two double-hemispherical condensers for electron detection. It has been brieffy discussed before (Merz et al 1992); in this section we present a more detailed description.

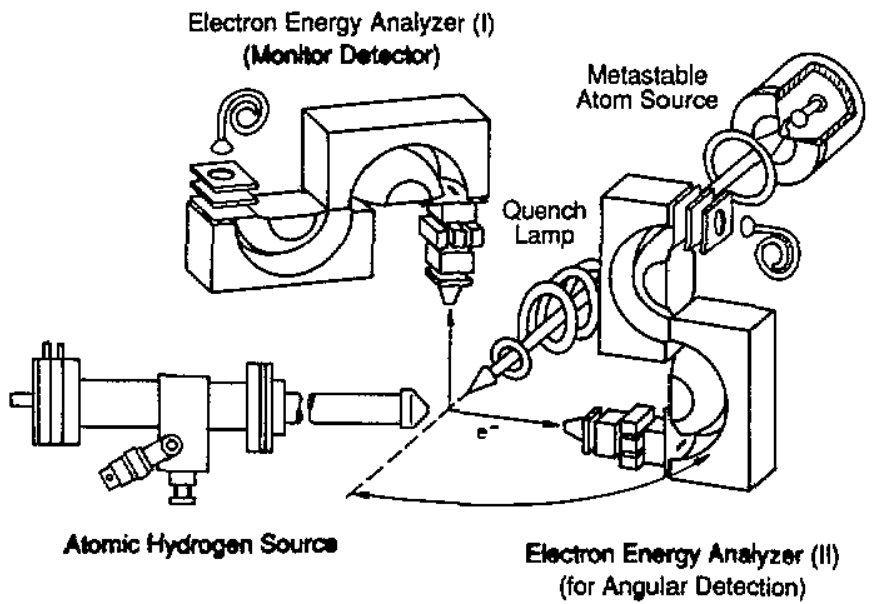

Figure 1. Semi-schematic illustration of the crossed-beam apparatus for the present study of angular- and energy-dependent electron spectra due to $\mathrm{He}^{*}\left(2^{1} \mathrm{~S}, 2^{3} \mathrm{~S}\right)+\mathrm{H}$ and $\mathrm{D}$ collisions. The experimental observation angle $\Theta$ of spectrometer ' $\mathrm{II}$ ' is related to the mean relative velocity vector $v_{\text {rel }}\left(\mathrm{He}^{*} \rightarrow \mathrm{H}_{/} \mathrm{D}\right)$ which is connected with the respective thermal velocity distributions of the atomic (molecular) beams. 


\subsection{Metastable atom beam}

The metastable helium atoms are produced in a differentially pumped cold cathode DC discharge $(I=10 \mathrm{~mA}, U=350-400 \mathrm{~V}$, exit hole $\varnothing=0.4 \mathrm{~mm})$, described earlier (Ruf et al 1987). Its flux can be monitored by a Faraday cup, utilizing electron emission from a stainless steel plate (electron emission coefficient assumed to be $\gamma=0.6$ (Dunning $e t$ al 1975). By a minor but efficient modification of the exit hole region, the $\mathrm{He}^{*}\left(2^{3} \mathrm{~S}\right)$ metastable flux could be increased from typically $7 \times 10^{14}$ atoms $/(\mathrm{s} \mathrm{sr})$ to $1.5 \times 10^{15}$ atoms/(s sr) (Merz 1993), leading to a density of about $2 \times 10^{7} \mathrm{~cm}^{-3}$ in the scattering region. The $\mathrm{He}^{*}$-beam is well collimated $(1: 120)$ and has a diameter of about $2 \mathrm{~mm}$ at the crossing point with the hydrogen beam. Our source design favours the production of $\mathrm{He}^{*}\left(2^{3} \mathrm{~S}\right)$ metastables, but the beam also contains a minor contribution (about $11 \%$ ) of $\mathrm{He}$ atoms in the $\left(2^{1} \mathrm{~S}\right)$ state. More than $99.5 \%$ of the latter can be removed via optical pumping using the irradiation from an air cooled helium DC discharge (see figure 1: 'quench-lamp') surrounding the He beam (Waibel et al 1988). The metastable He beam has supersonic character and its velocity density distribution can be described by:

$$
f(v)=\text { const } v^{2} \exp \left[-m(v-u)^{2} /(2 k T)\right]
$$

with $T \simeq 20 \mathrm{~K}, u \simeq 1650 \mathrm{~m} \mathrm{~s}^{-1}$. The average velocity of the metastable atoms is then $\bar{v}_{\mathrm{He}} \simeq 1750 \mathrm{~m} \mathrm{~s}^{-1}$, and the distribution has a width (FWHM) of $\Delta v / \bar{v}_{\mathrm{He}} \simeq 30 \%$.

\subsection{Atomic hydrogen beam}

The hydrogen/deuterium atoms are generated in an air cooled microwave discharge (2.45 $\mathrm{GHz}$, typical input power $150 \mathrm{~W}$, pressure in the cavity $0.5 \mathrm{mbar}$ ) outside the vacuum chamber and guided to the reaction zone by a PTFE-covered stainless steel tube. The source consists of a triple glass tube system: the hydrogen/deuterium discharge burns in the inner tube $(\varnothing=10 \mathrm{~mm})$ while the outer two tubes serve as a cooling circuit. Before use the discharge tube is treated with $\mathrm{HF}$ acid to enlarge the dissociation ratio. For the discharge region itself we estimate this ratio to be larger than $n(\mathrm{H}, \mathrm{D}) / n\left(\mathrm{H}_{2}, \mathrm{D}_{2}\right) \geqslant 0.9$. During operation impurities cover the surface of the discharge tube and continuously reduce the dissociation rate. By recombination at the surface of the PTFE tube $(l=50 \mathrm{~cm}, \varnothing=9.6 \mathrm{~mm})$, the flux of $H / D$ reaching the reaction centre is further decreased by about a factor of two. Averaged over a typical span of an experiment $(48 \mathrm{~h})$, the experimentally determined density ratio is $n(\mathrm{H}, \mathrm{D}) / n\left(\mathrm{H}_{2}, \mathrm{D}_{2}\right) \approx$ $0.2-0.3$ (Merz 1993). The H/D beam is not collimated, the PTFE-covered stainless steel tube simply ends in a 'nozzle' $(\varnothing=1 \mathrm{~mm})$ at a distance of $5 \mathrm{~mm}$ from the scattering centre. The density in the scattering volume can be estimated to be $n_{\mathrm{H} / \mathrm{D}} \simeq 3 \times 10^{11} \mathrm{~cm}^{-3}$. The velocity distributions of the $H / D$ atom beams were not measured during this experiment, but can be assumed to be Maxwell distributions for an effusive quasi-beam of $T=300 \mathrm{~K}$. Together with the velocity distribution of the $\mathrm{He}^{*}$-beam this leads to collision energy distributions with average values close to $\vec{E}_{\mathrm{kin}} \approx 50 \mathrm{meV}$ for both isotopes. The respective distributions peak at $E_{\mathrm{ks}} \approx 30 / 35 \mathrm{meV}$ and their widths (FWHM) are $\Delta E_{\mathrm{kin}} \approx 49 / 42 \mathrm{meV}$.

\subsection{Electron spectrometers}

Figure 1 shows two double-hemispherical condensers, used to measure electron as well as ion energy spectra. In this paper, we restrict the discussion to the electron data. One 
of the two spectrometers (I) detects charged particles at $90^{2}$ with respect to both atomic beam axes and serves as a monitor in the determination of angle-dependent, energyintegrated cross sections. Its detection geometry corresponds to the one always used in previous work (Hotop ct al 1971, Morgner and Niehaus 1979, Waibel et al 1988). It is placed on top of the reaction chamber (not shown in figure 1) with the entrance hole of its electron optics $25 \mathrm{~mm}$ above the crossing point of the atomic beams. The second spectrometer (II) is rotatable and detects electrons ejected in the collision plane spanned by the atomic beams; its entrance hole is located $60 \mathrm{~mm}$ from the scattering centre. The detection angle $\Theta$ in the plane of the atomic beams is defined with regard to the relative velocity vector $v_{\text {rel }}\left(\mathrm{He}^{*} \rightarrow \mathrm{H}, \mathrm{D}\right)$, with the He velocity vector in the centre-ofmass frame defining the forward direction.

Both detectors are operated at the fixed transmission energy of $4.8 \mathrm{eV}$ and have a nominal energy resolution of $\Delta \varepsilon_{\text {res }}=0.5 \%$. Over the total accumulation time of a typical spectrum $(48 \mathrm{~h})$ the effective energy resolution amounts to $\Delta \varepsilon_{\mathrm{Sp}}(\mathrm{H} / \mathrm{D})=25 / 30 \mathrm{meV}$ and $\Delta \varepsilon_{\mathrm{Sp} 11}(\mathrm{H} / \mathrm{D})=30 / 35 \mathrm{meV}$. These values were experimentally determined through evaluation of the widths of simultaneously measured photoelectron peaks due to the reaction $\mathrm{H}_{2}+\mathrm{He}(\mathrm{I} \alpha)$. (The He resonance photons are also produced in the metastable atom source).

The angular resolution of the present measurements is rather poor; it is mainly limited by the velocity spread of both atomic beams $\left(\rightarrow \Delta \Theta \approx \pm 26^{\circ}\right)$, but also by the angular spread of the non-collimated hydrogen/deuterium beam. The effective angular resolution amounts to $\Delta \Theta_{\text {res }} \approx \pm 37^{\circ}$ (Merz 1993). (The geometrical angular resolution of the spectrometer, i.e. the acceptance angle, is $\pm 3^{\circ}$.)

With regard to the work of Waibel et al (1988), we have modified the electron optics of the two spectrometers. Previously, the voltages applied to the different elements of the optics were chosen proportional to the pass energy. This led to an energy dependent compression of the electron energy scale which had to be corrected for afterwards. In the present study the incoming electron flux is accelerated/retarded to the pass energy of $4.8 \mathrm{eV}$ and the voltages applied to the optics are held fixed. In this operation mode no energy-dependent scaling effects were observed.

Due to the different distances between the entrance slits of the electron optics of the detectors and the scattering volume, the resulting energy-dependent transmissions are also different. While for spectrometer II the transmission is more or less constant $(\simeq 10 \%)$ over the relevant energy range between $3 \mathrm{eV}$ and $8 \mathrm{eV}$, the transmission of detector I has a maximum at $\varepsilon=3 \mathrm{eV}$ and decreases for higher energies by about $10 \% \mathrm{eV}^{-1}$. The transmission functions were continuously controlled by recording photoelectron spectra of the process $\mathrm{O}_{2}+\mathrm{He}(\mathrm{I} \alpha)$ and comparing the measured intensities with literature data (see appendix).

A double $\mu$-metal shielding was used to reduce surrounding magnetic fields. The residual field in the collision region and at the two spectrometers had values smaller than $5 \times 10^{-8} \mathrm{~T}$ in vertical and horizontal direction (Merz 1993).

\section{Results and discussion}

\subsection{Data processing}

In order to establish well defined experimental spectra, the measured raw data were evaluated and corrected as follows: the energy scale was calibrated properly and the 
spectra were corrected for the energy dependence of the spectrometer transmission (see appendix). Next, the data sets were corrected for contributions from $\mathrm{He}^{*}+\mathrm{H}_{2} / \mathrm{D}_{2}$ collisions. As mentioned above, the hydrogen beam did not only contain atomic but also molecular hydrogen to a considerable extent. Therefore the raw data include nonnegligible portions of electrons due to autoionizing collisions between metastable helium atoms and molecular hydrogen/deuterium. In spite of the rather low dissociation rate, this contribution is still comparatively small since at thermal energies the (total) Penning ionization cross section for $\mathrm{He}^{*}\left(2^{3} \mathrm{~S}\right)+\mathrm{H}_{2}$ collisions is more than an order of magnitude smaller than the corresponding $\mathrm{He}^{*}\left(2^{3} \mathrm{~S}\right)+\mathrm{H}$ cross section $\left(\sigma_{\mathrm{H}_{2}}\left(E_{\text {kin }}=\right.\right.$ $50 \mathrm{meV})=1.89 \times 10^{-16} \mathrm{~cm}^{2}, \sigma_{\mathrm{Hx}}\left(E_{\mathrm{k} \text { । }}=50 \mathrm{meV}\right)=2.85 \times 10^{-15} \mathrm{~cm}^{2}$ (Barnett 1990, Siska 1993).

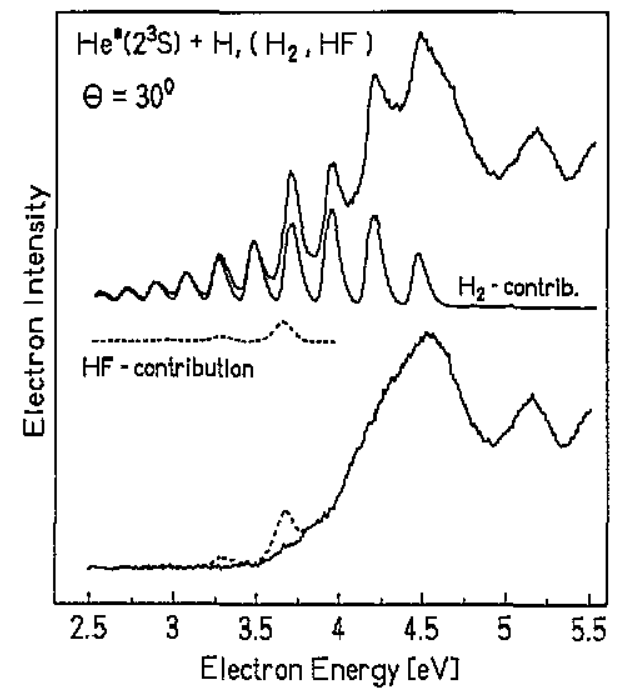

Figure 2. Experimental electron-energy spectra for the systems $\mathrm{He}^{*}\left(2^{3} \mathrm{~S}\right)+\mathrm{H}, \mathrm{H}_{2}$ and $\mathrm{HF}$ at $\Theta=30^{\circ}$. The spectra due to $\mathrm{He}^{*}\left(2^{3} \mathrm{~S}\right)+\mathrm{H}_{2}$ and $\mathrm{HF}$ (broken curve) were subtracted from the raw data set (top spectrum), leading to a pure $\mathrm{He}^{*}\left(2^{3} \mathrm{~S}\right)+\mathrm{H}$ spectrum (bottom).

The upper part of figure 2 shows a raw spectrum for the detection angle $\Theta=30^{\circ}$ together with a pure $\mathrm{He}^{*}\left(2^{3} \mathrm{~S}\right)+\mathrm{H}_{2}$ spectrum taken at the same laboratory angle. After subtraction of the $\mathrm{H}_{2}$ part weak features remained on the low energy side of the main peak located at $\varepsilon_{\text {rel }} \approx 4.5 \mathrm{eV}$. This structure was traced to collisions between $\mathrm{He}^{*}$-atoms and HF. The hydrogen fluoride most probably stems from the interaction of the $\mathrm{H} / \mathrm{H}_{2}$-plasma with the PTFE-tube which ends close to the plasma region. The electron spectrum used to correct the present data was measured by Yencha et al (1993). A corresponding $\mathrm{He}^{*}\left(2^{3} \mathrm{~S}\right)+\mathrm{DF}$ spectrum was constructed by scaling the $\mathrm{HF}$ spectrum according to the vibrational spacing of the $\mathrm{HF}^{+} / \mathrm{HD}^{+}$potential curves (Huber and Herzberg 1979).

Our study shows that the electron-spectroscopic results depend on the detection angle. Not only the spectra due to $\mathrm{He}^{*}\left(2^{3} \mathrm{~S}\right)+\mathrm{H} / \mathrm{D}$ collisions, but also the processes $\mathrm{He}^{*}\left(2^{3} \mathrm{~S}\right)+\mathrm{H}_{2} / \mathrm{D}_{2}$ - especially for deuterium-turned out to be angle-dependent. As known for other repulsive Penning ionization systems (Niehaus 1981, Mitsuke et al 1989), the energy integrated cross sections for the processes $\mathrm{He}^{*}\left(2^{3} \mathrm{~S}\right)+\mathrm{H}_{2} / \mathrm{D}_{2}$ peak in the backward direction of the collision system $\left(\left\langle\sigma_{\mathrm{H}_{2}}\left(\Theta=180^{\circ}\right)\right\rangle /\left\langle\sigma_{\mathrm{H}_{2}}\left(\Theta=0^{\circ}\right)\right\rangle \approx 3.3\right.$; 


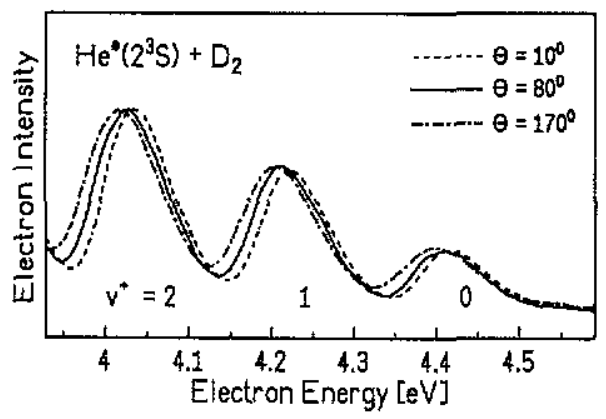

Figure 3. Selected energy range of angle-differential electron spectra due to $\mathrm{He}^{*}\left(2^{3} \mathrm{~S}\right)+\mathrm{D}_{2}$ collisions. The peaks, labelled $v^{+}=0-2$, are due to transitions to different vibrational states in the ionic exit channel potential $\mathrm{He}+\mathrm{D}_{2}^{+}$. The angle $\Theta$ is-in this case-related to the mean relative velocity vector of $\mathrm{He}^{*}+\mathrm{D}_{2}$ collisions.

$\left.\left\langle\sigma_{\mathrm{L}_{2}}\left(\Theta=180^{\circ}\right)\right\rangle /\left\langle\sigma_{\mathrm{D}_{2}}\left(\Theta=0^{\circ}\right)\right\rangle \approx 2.5\right)$. In figure 3 we compare three spectra for the system $\mathrm{He}^{*}\left(2^{3} \mathrm{~S}\right)+\mathrm{D}_{2}$ for the detection angles $\Theta=10^{\circ}, 80^{\circ}$ and $170^{\circ}$ (here $\Theta$ is relative to $v_{\mathrm{rel}}\left(\mathrm{He}^{*} \rightarrow \mathrm{D}_{2}\right)$ ). Note that these angles correspond to $\Theta=0^{\circ}, 90^{\circ}$ and $180^{\circ}$ in the $\mathrm{He}^{*}+\mathrm{D}$ system. The data sets reveal a variation with detection angle not only for the energy position but also for the shape of the peaks, which are due to transitions to different vibrational levels $\left(v^{+}=0-2\right)$ in the exit channel potential. The energy calibration of these spectra is based on the known centre-of-mass energies of the photoelectron peaks from the reaction $\mathrm{D}_{2}(v=0)+\mathrm{He}(\mathrm{I} \alpha) \rightarrow \mathrm{D}_{2}^{*}\left(v^{+}=1-5\right)+\mathrm{e}^{-}(\varepsilon)$ (these are weakly present at higher energies not included in figure 3 ). In this way the angle-dependent Doppler shift of the pholoelectron peaks $\left(\Delta \varepsilon_{\mathrm{D}} \leqslant 12 \mathrm{meV}\right.$ at $\left.\varepsilon=5 \mathrm{eV}\right)$ is taken into account.

In contrast to earlier work (Waibel et al 1988), we found no indications for any contribution due to vibrationally excited $\mathrm{H}_{2} / \mathrm{D}_{2}(v>0)$ in the $\mathrm{He}^{*}\left(2^{3} \mathrm{~S}\right)+\mathrm{H} / \mathrm{D}+\mathrm{H}_{2} / \mathrm{D}_{2}$ electron energy spectra. We therefore believe that molecules which leave the dissociation region in a vibrationally excited state relax when passing the PTFE tube.

\section{2. $H e^{*}\left(2^{3} S\right)+H\left(l^{2} S\right)$}

Figure 4 shows the relevant potential curves for the system $\mathrm{He}^{*}\left(2^{1} \mathrm{~S}, 2^{3} \mathrm{~S}\right)+$ $\mathrm{H}\left(1^{2} \mathrm{~S}\right) \rightarrow \mathrm{He}+\mathrm{H}^{+}+\mathrm{e}^{-}$, as discussed in detail by Movre and Meyer (1994). The $\mathrm{He}^{*}\left(2^{3} \mathrm{~S}_{1}\right)+\mathrm{H}\left(1^{2} \mathrm{~S}_{1 / 2}\right){ }^{2} \Sigma^{+}$entrance channel potential has a well depth of $2.284 \mathrm{eV}$ at the internuclear distance $R=3.42 a_{0}$. The ionic potential has a well depth of $2.040 \mathrm{eV}$ at $R=1.463 a_{0}$ (Kolos and Peek 1976). The inset illustrates the $R$-dependence of the imaginary part of the entrance channel potential, i.e. the total autoionization width $\Gamma(R)$. We have omitted the (basically repulsive) ${ }^{4} \Sigma^{+}$potential curve since it is not involved in the autoionization process as a result of spin conservation (see, e.g., Gray et al 1985).

Since the electron energy spectrum is closely related to the difference potential of entrance and exit channel potentials $\left(\varepsilon(R)=V^{*}(R)-V^{+}(R)\right.$ (Miller 1970)), the spectra due to $\mathrm{He}^{*}\left(2^{3} \mathrm{~S}_{1}\right)+\mathrm{H}\left(1^{2} \mathrm{~S}_{1 / 2}\right)$ collisions have a spread in energy over more than three electron volts. The electron energy spectrum of the process $\mathrm{He}^{*}\left(2^{3} \mathrm{~S}_{1}\right)+\mathrm{H}\left(1^{2} \mathrm{~S}_{1 / 2}\right)$ has already been studied in detail by several authors (Hotop et al 1971, Morgner and Niehaus 1979, Waibel et al 1988), but in contrast to the present work the spectrum was always measured at a fixed angle, perpendicular to the crossed atomic beams. Previously, 


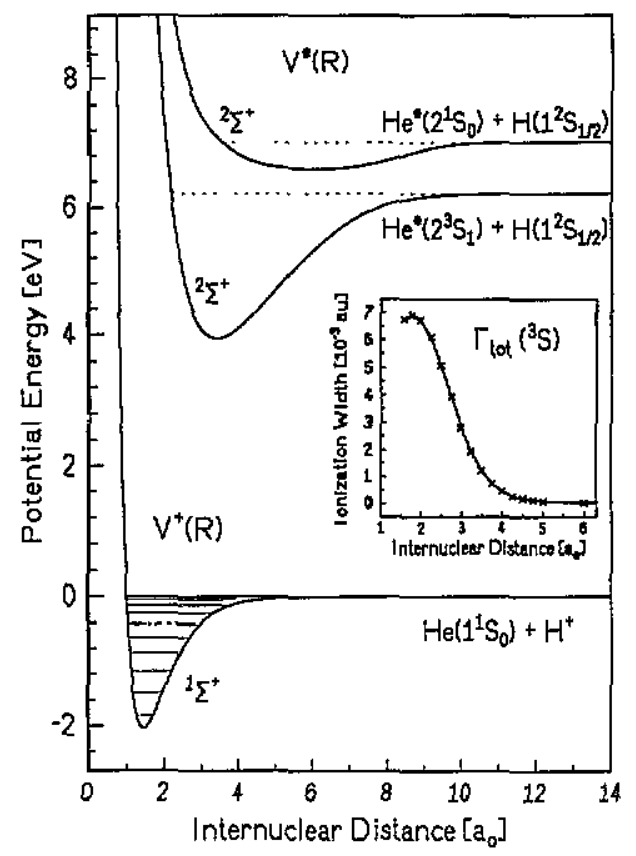

Figure 4. Potential energy curves of the relevant entrance and exit channel electronic states, according to Movre and Meyer (1994). The inset shows the $R$-dependence of the total autoionization width for $\mathrm{He}^{*}\left(2^{3} \mathrm{~S}\right)+\mathrm{H}$ collisions (for details see Movre and Meyer 1994).

this fundamental Penning ionization system was believed to yield an isotropic electron energy spectrum (Morgner and Niehaus 1979, Waibel et al 1988). It was generally assumed that strong attraction, leading to spiralling trajectories, averages out effects associated with a possible anisotropic electron emission in the molecule-fixed frame, justifying a neglect of electron angular distribution effects (Morgner and Niehaus 1979, Niehaus 1981, Lorenzen et al 1983, Waibel et al 1988). An additional argument for the neglect of an angular-dependence in the $\mathrm{He}^{*}\left(2^{3} \mathrm{~S}\right)+\mathrm{H}$ electron energy spectrum was based on the assumption that the outgoing electron is-due to the underlying exchange mechanism-emitted from the $\mathrm{He}^{*}$-atom, close to the centre-of-mass of the system. Therefore, there should be little reason for energy and angular momentum exchanges due to a recoil effect (Lorenzen et al 1983, Waibel et al 1988), as discovered for the Penning ionization system $\mathrm{He}^{*}\left(2^{3} \mathrm{~S}\right)+\mathrm{Li}($ Merz et al 1989, 1990).

Before we discuss the angle dependent spectra let us first compare our $\Theta=90^{\circ}$ spectrum with earlier results. We restrict this discussion to the spectrum of Waibel et al (1988) since it has better statistics and energy resolution than the earlier spectra of Hotop et al (1971) and of Morgner and Niehaus (1979). Figure 5 contains three spectra: the spectrum by Waibel et al at the top and two data sets of the present work, measured with the two different spectrometers (see figure 1) in plane and perpendicular, respectively. Both spectra were measured at $\Theta=90^{\circ}$, the one labelled ' $T$ ' at a position perpendicular to the plane of the atomic beams and therefore automatically perpendicular to the relative velocity vector. The other one ('II') was taken in plane, but also perpendicular to the relative velocity vector.

The spectrum of Waibel et al (1988) shows some artificial structures on the low energy side of the main Airy peak (at energies close to $4.5 \mathrm{eV}$ ) which result from a nonperfect correction for the $\mathrm{H}_{2}$ contributions (Waibel 1986, Waibel et al 1988). The 


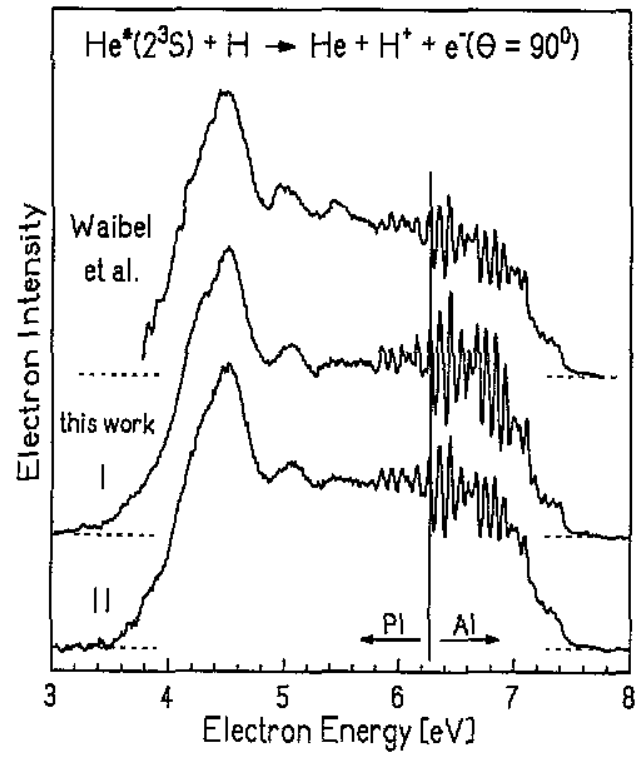

Figure 5. Electron energy spectra for the collision complex $\mathrm{He}^{*}\left(2^{3} \mathrm{~S}\right)+\mathrm{H}$ measured at $\Theta=$ $90^{\circ}$ : 'Waibel et al': taken from Waibel et al (1988); this work : I, measured with the monitor detector out of plane; II, measured with the spectrometer for angular detection in the plane of the two atomic beams (see figure 1).

oscillatory structure forming the low energy part of the spectrum is often called 'Airyoscillation', since in the semi-classical theory this part of the electron energy spectrum is given by the square of an Airy function (Miller 1970). In the region of the two supernumerary peaks at energies close to $4.9 \mathrm{eV}$ and $5.4 \mathrm{eV}$ there is some further structure in the spectrum by Waibel et al which is not present in the new data sets. These features can be traced to an incomplete subtraction of contributions due to collisions between $\mathrm{He}^{*}\left(2^{3} \mathrm{~S}\right)$ and vibrationally excited $\mathrm{H}_{2}$. We also note that the overall shape of Waibel's spectrum deviates from the present transmission-corrected data which were obtained with spectrometers I and II and are found to be in good overall agreement. The ratio of Penning and associative ionization is nearly the same in the two present spectra while in the spectrum by Waibel et $a l$ the main Airy peak is more strongly pronounced in comparison with the associative ionization part. A closer inspection of Waibel's transmission correction revealed that, for energies larger than $5 \mathrm{eV}$, the transmission was actually smaller than assumed (Waibel 1986). This, of course, influenced the result of the previous fit calculation which was carried out in order to get more information on the $R$-dependence of the autoionization width. Especially the sharp bending of the autoionization width function $\Gamma(R)$ of Waibel et al $(1988$, see also Merz et al 1990) at distances close to $R=3 a_{0}$ from exponential to constant is in part a consequence of the inadequate transmission correction. Apart from that, the results of the former fit suffered from the incorrect assumption that the process can be described in a simplified, angle-independent way. We emphasize, however, that this assumption does not affect the relative size of the calculated partial cross sections $\sigma_{v J}$, nor the interpretation of the associative and quasi-associative ionization structure (Waibel et al 1988).

We note that some differences between the present spectra I and II are visible, especially in the associative ionization part. The fine oscillations in the Al regime 
$(\varepsilon \geqslant 6.27 \mathrm{eV}$ ) result from transitions into bound ro-vibrational states in the exit-channel. The sum over all contributing partial waves, convoluted with the experimental energy resolution, leads to the observed structure. The modulation of the structures is stronger in spectrum I which was measured out of plane. This also holds for the fine structures in the high-energy part of the PI-region at energies $5.8 \mathrm{eV}<\varepsilon<6.27 \mathrm{eV}$ which are usually labelled "quasi-associative" ionization, since these peaks are due to transitions into highlying, rotationally-bound resonance states which decay to $\mathrm{He}+\mathrm{H}^{+}$and lie in the energy regime of PI. In both cases the difference in modulation is mainly due to the better angular resolution for the data of spectrometer $I$ which detects only electrons emitted perpendicular to the plane defined by the atomic beams. The electrons detected by spectrometer II, however, correspond to an average over the different relative velocity directions $\left( \pm 26^{\circ}\right)$ associated with the angular and velocity spread of the hydrogen/ deuterium beam; as a consequence, some of the structure is smeared out. There are some minor differences in the Penning ionization part as well. In view of the high statistical accuracy in spectrum I, i.e. 100 kcounts in the maximum of the Airy peak at $4.5 \mathrm{eV}$ (spectrum II: $10 \mathrm{kcounts),} \mathrm{we} \mathrm{can} \mathrm{be} \mathrm{sure} \mathrm{that} \mathrm{even} \mathrm{the} \mathrm{slight} \mathrm{shoulder} \mathrm{in} \mathrm{the}$ main Airy peak at $4.3 \mathrm{eV}$ is significant (see figure 6 below).

The angle-dependent spectra due to the autoionization process $\mathrm{He}^{*}\left(2^{3} \mathrm{~S}\right)+\mathrm{H}\left(1^{2} \mathrm{~S}\right)$ were already discussed in some detail in preceding publications (Merz et al 1992, 1993). Methodology and details of the $a b$ initio calculations are fully described by Movre and Meyer (1994). Here we include these results for completeness. In short the theoretical spectra are derived from the general double-differential cross sections $d^{2} \sigma /(d \varepsilon d \Theta)$ for autoionization from the metastable entrance to the ionic exit channel, as given e.g. by Nakamura (1969) and Bieniek (1978), involving a fully quantum mechanical treatment of heavy particle and electron motions including their coupling without any further approximations than those inherent in the local complex potential approximation. Note, in particular, that initial and final state wavefunctions do not need to have the same heavy particle angular momentum. The heavy particle wavefunctions are coupled by $V_{l}(R)$, the complex partial wave components of the electronic coupling matrix element, where $l$ is the electron angular momentum with regard to the centre-of-mass of the quasi-molecule and the electron energy dependence of the coupling is removed by the resonance condition $\varepsilon(R)=V^{*}(R)-V^{+}(R)$. In terms of $V_{l}(R)$, the total autoionization width $\Gamma(R)$ is given by $\Gamma(R)=2 \pi \Sigma_{l}\left|V_{l}(R)\right|^{2}$. The coupling elements are obtained in a two-step procedure by first casting the resonance-continuum coupling into a compact one-electron function and then projecting the latter onto the solutions of a staticexchange electron scattering calculation (Movre and Meyer 1994).

Figure 6 shows a comparison between theory and experiment for three detection angles: $\Theta=10^{\circ}, 90^{\circ}$ and $180^{\circ}$. Due to the broad collision energy distribution, the experimental spectra taken under a specific detection angle (with respect to the mean relative velocity vector) average over a rather large range of emission angles (with respect to the actual relative velocity vector). The theoretical spectra, calculated for a grid of 25 collision energies and 13 emission angles and convoluted with the experimental electron energy resolution, are therefore weighted according to the energy distributions which are appropriate for the different emission angles that contribute to a particular detection angle. The divergence of the H-beam, however, has been neglected, and that may well be one reason for the remaining discrepancies between theoretical and experimental spectra. We interpret the very good overall agreement between the theoretical and the experimental results as a strong support for the validity of the local complex potential approach in the frame of the Born-Oppenheimer approximation. 


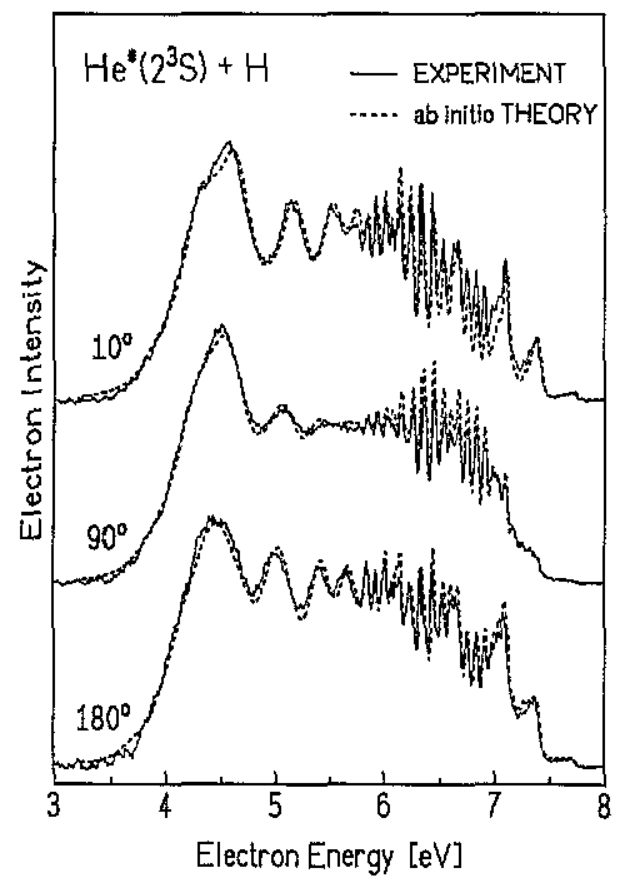

Figure 6. Comparison between experimental (full curve) and theoretical (broken curve) electron-energy spectra for the detection angles $\Theta=10^{\circ}, 90^{\circ}$ and $180^{\circ}$, respectively (Merz et al 1993). The experimental spectra have a common intensity scale proportional to the cross section. The experimental data were individually adjusted to the theoretical spectra at the different detection angles.

\section{3. $H e^{*}\left(2^{3} S\right)+D\left(I^{2} S\right)$}

To complement our understanding of simple Penning ionization systems we have extended our study of angle-dependent spectra to collisions between metastable $\mathrm{He}$ atoms with deuterium atoms. Figure 7 compares two spectra from the processes $\mathrm{He}^{*}\left(2^{3} \mathrm{~S}\right)+\mathrm{H}\left(1^{2} \mathrm{~S}\right)$ and $\mathrm{D}\left(1^{2} \mathrm{~S}\right)$ for perpendicular electron detection $\left(\Theta=90^{\circ}\right)$. It turns out that most of the structure of the $\mathrm{H}$ spectrum is also present, but less pronounced in the D spectrum, both in the AI-part and in the PI-regime. This is mainly due to the larger number of contributing heavy particle partial waves $J^{*}$. A classical estimate for the average collision energy $\bar{E}_{\text {kin }}=50 \mathrm{meV}$ yields $J_{\max }^{*}=26 \hbar$ as the maximal angular momentum that contributes to ionization in $\mathrm{He}^{*}\left(2^{3} \mathrm{~S}\right)+\mathrm{H}$ collisions, while for the process $\mathrm{He}^{*}\left(2^{3} \mathrm{~S}\right)+\mathrm{D}$ the corresponding number is $J_{\max }^{*}=34 \hbar$. Moreover, the ro-vibrational levels of $\mathrm{HeD}^{+}$are more closely spaced than those of $\mathrm{HeH}^{+}$. The difference in reduced masses affects most strongly the interference between the so-called fast oscillations (Tellinghuisen 1985, Müller et al 1987, 1991) and the Airy oscillation, which accounts for the observed changes in the structure of the PI part, e.g. for the disappearance of the shoulder in the main Airy peak of the $\mathrm{H}$ spectrum at $\varepsilon=4.3 \mathrm{eV}$.

Apart from that, the two spectra in figure 7 show similar features: in the AI part one observes characteristic steps (at energies $E_{\mathrm{el}} \simeq 7.55 \mathrm{eV}, 7.3 \mathrm{eV}$ and $7.1 \mathrm{eV}$ for D), which are due to the opening of vibrational channels $\left(v^{+}=1-3\right)$ in the exit potential, as already discussed for the $\mathrm{H}$ spectra (Merz et al 1992, 1993). The secondary Airy peaks at energies $E_{\mathrm{el}}=5 \mathrm{eV}$ and $5.5 \mathrm{eV}$, respectively, are only weakly visible in the D 


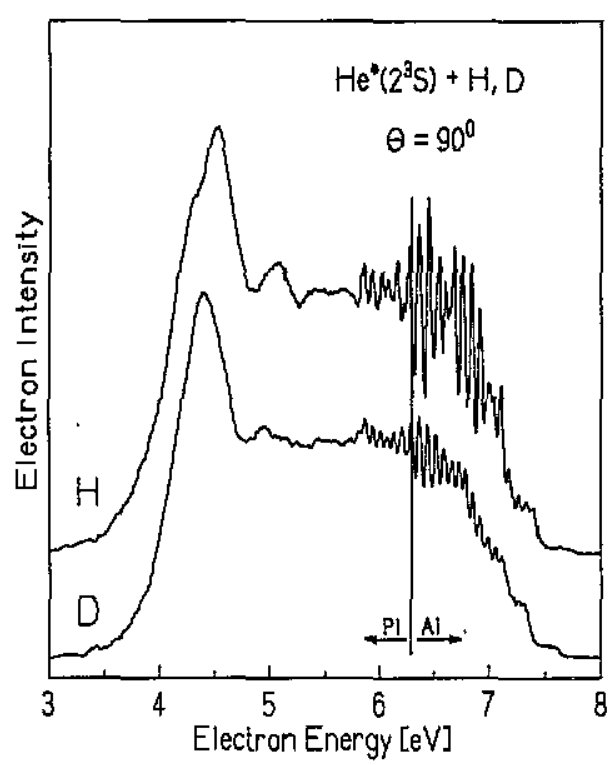

Figure 7. Energy spectra at $\Theta=90^{\circ}$ for $\mathrm{He}^{*}\left(2^{3} \mathrm{~S}\right)+\mathrm{H}$ and $\mathrm{D}$ collisions, illustrating the influence of the different target masses on the modulation structures in the Penning and associative ionization spectrum. Both spectra were measured with approximately the same electron energy resolution of $\Delta \varepsilon \approx 30 \mathrm{meV}$.

spectrum at this detection angle. However, for other detection angles they show a similar angular behaviour as observed in the $H$ spectra (see figures 6 and 8 ).

Figure 8 shows $\mathrm{He}^{*}\left(2^{3} \mathrm{~S}\right)+\mathrm{D}$ spectra for seven detection angles between $\Theta=0^{\circ}$ and $180^{\circ}$. In general their angle-dependent behaviour is similar to that of the $\mathrm{He}^{*}\left(2^{3} \mathrm{~S}\right)+\mathrm{H}$ spectra: the $\mathrm{D}$ spectra show clearly that most of the features, which are strongly modulated in forward and backward direction, such as the steps in the AI regime or the secondary Airy peaks, are averaged out at angles close to $90^{\circ}$. As for $\mathrm{He}^{*}\left(2^{3} \mathrm{~S}\right)+\mathrm{H}$, the energy position of the secondary Airy maxima is angle dependent: one notices a continuous shift of up to $150 \mathrm{meV}$ from higher to lower energies when going from $\Theta=$ $0^{\circ}$ to $180^{\circ}$. The spacing between the secondary Airy structures should behave like $1 / \sqrt{\mu}$ with $\mu$ representing the reduced mass (Tellinghuisen 1985). In fact a comparison between figures 6 and 8 shows that-for example in the backward direction-there are three secondary Airy peaks present in the $\mathrm{H}$ spectrum while in the corresponding $\mathrm{D}$ spectrum four maxima appear, in accordance with $\sqrt{\mu_{\mathrm{D}} / \mu_{\mathrm{H}}}=1.29$. The quasi-associative ionization structures at energies between 5.8 and $6.2 \mathrm{eV}$ are, as for $\mathrm{He}^{*}\left(2^{3} \mathrm{~S}\right)+\mathrm{H}$, substantially stronger in forward and backward direction of the collision system than in the perpendicular direction.

The observed angular variation of the electron spectra can be interpreted in terms of the anisotropy of the internal angular distribution (IAD) for electron emission from the quasi-molecule (HeD)* (Ebding and Niehaus 1974, Micha and Nakamura 1975, Morgner 1978, Hoffmann and Morgner 1979, Niehaus 1981, Mitsuke et al 1989, Merz et al 1992, 1993), i.e. the angle-dependent electron intensity as emitted in the frame of the autoionizing coilision complex at a given internuclear separation $R$ :

$$
I_{\mathrm{IAD}}(\vartheta, R)=\left|\sum_{l} \mathrm{i}^{-l} Y_{\mathfrak{N}}(\vartheta) V_{l}(R)\right|^{2}
$$




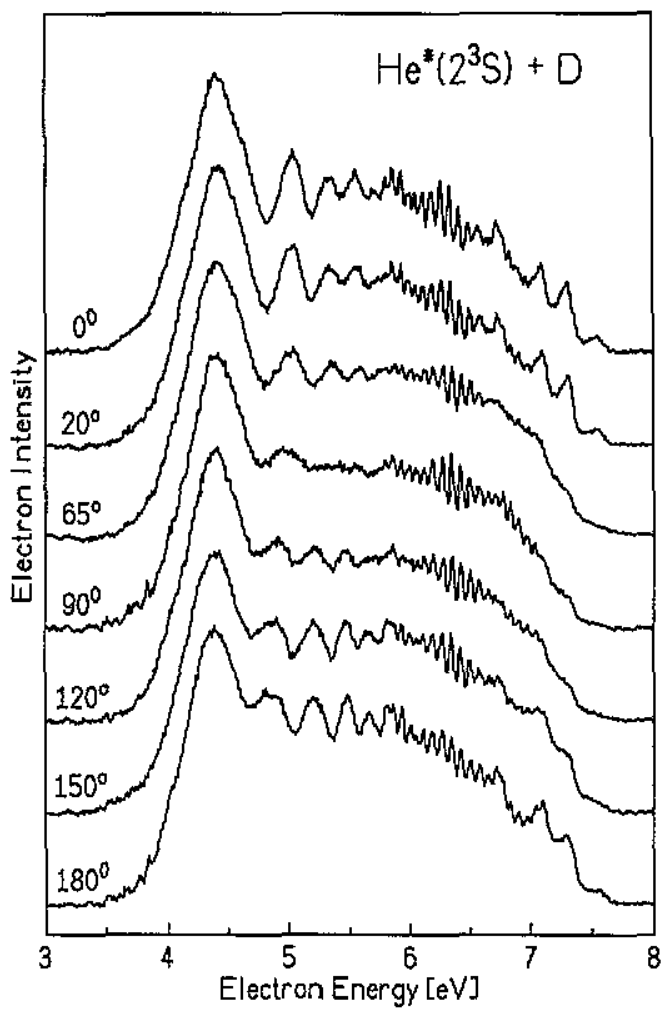

Figure 8. Angle-differential experimental study of the system $\mathrm{He}^{*}\left(2^{3} \mathrm{~S}\right)+\mathrm{D}$ for seven different detection angles referring to $v_{\mathrm{rel}}\left(\mathrm{He}^{*} \rightarrow \mathrm{D}\right)$.

where $\vartheta$ represents the body-fixed electron emission angle. The amplitudes $V_{l}(R)$ are referred to the centre-of-mass of the collisional complex. Figure 9 displays the $a b$ initio results for the quantities $V_{l}(R)$ of $\mathrm{He}^{*}\left(2^{3} \mathrm{~S}\right)+\mathrm{H} / \mathrm{D}$. The IAD visualizes the consequences of the phase relations of the coupling elements $V_{l}(R)$ at a particular distance $R$, but note that the strong $R$ dependence of $V_{l}(R)$, shown in figure 9 , is also important because the Airy peak structure is due to interference of transition amplitudes related to different distances $R$ for which the difference potential matches. A full account of the computational procedures is given by Movre and Meyer (1994). The slight differences between the two isotopic systems are entirely due to the different locations of the corresponding centres-of-mass. Figure 10 shows the results of the $a b$ initio calculation of the IAD for small (top: $2.0 a_{0} \leqslant R \leqslant 3.5 a_{0}$ ) and intermediate (bottom: $3.75 a_{0} \leqslant R \leqslant 5 a_{0}$ ) internuclear distances. For small distances strong forward and backward maxima exist, while for larger distances there is almost no backward peak left, and the forward and perpendicular intensities are nearly equal.

This behaviour can be partly correlated with the electronic structure of the resonance state. At short distances $\left(R<3.5 a_{0}\right)$ the resonance state is well described by a single configuration $1 \sigma(2 \sigma)^{2}$ where the $2 \sigma$ orbital resides predominantly at $\mathrm{H}$. For intermediate distances the electronic structure gradually changes to the configurations $1 \sigma^{1}(2 \sigma, 3 \sigma)$ and then ${ }^{3}(1 \sigma, 2 \sigma) 3 \sigma$ where $2 \sigma$ and $3 \sigma$ correlate to $2 s$ of He and $1 \mathrm{~s}$ of $H$, respectively (Movre and Meyer 1994). 
$H e^{*}\left(2^{3} \mathrm{~S}\right)+\mathrm{H}$

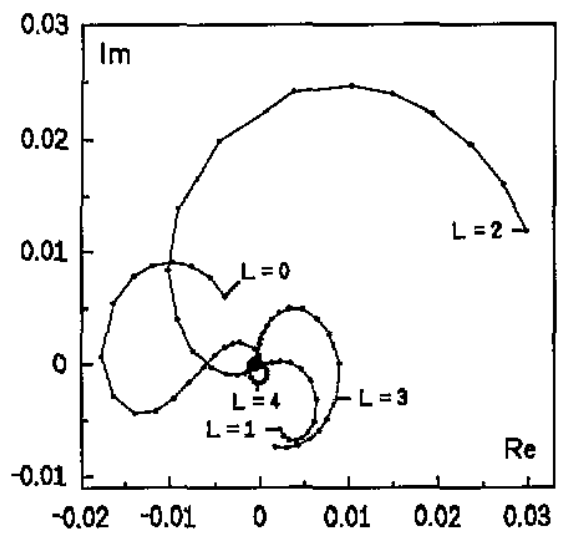

$H e^{*}\left(2^{3} s\right)+D$

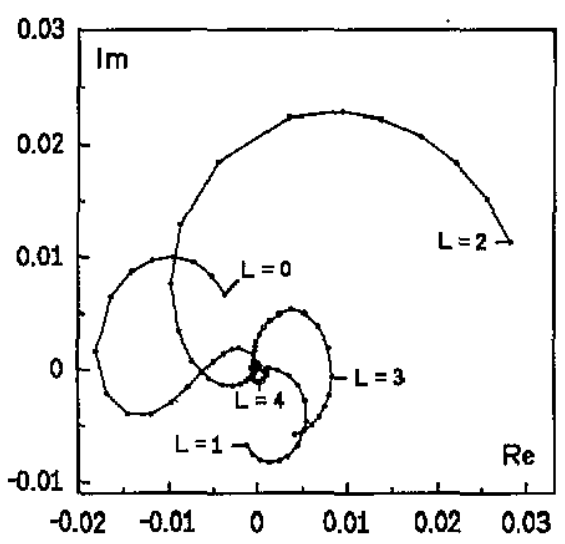

Figure 9. Complex partial wave components of the electronic coupling matrix element $V(R)(a u)$, which depends on the internuclear distance and the electron angular momentum $l$, for the systems $\mathrm{He}^{*}\left(2^{3} \mathrm{~S}\right)+\mathrm{H}, \mathrm{D}$ (see text). Points are at $R=1.6 \rightarrow 2.1(\Delta R=0.1), 2.25 \rightarrow 5$ $(0.25), 6 \rightarrow 10(1)(2 u)$.
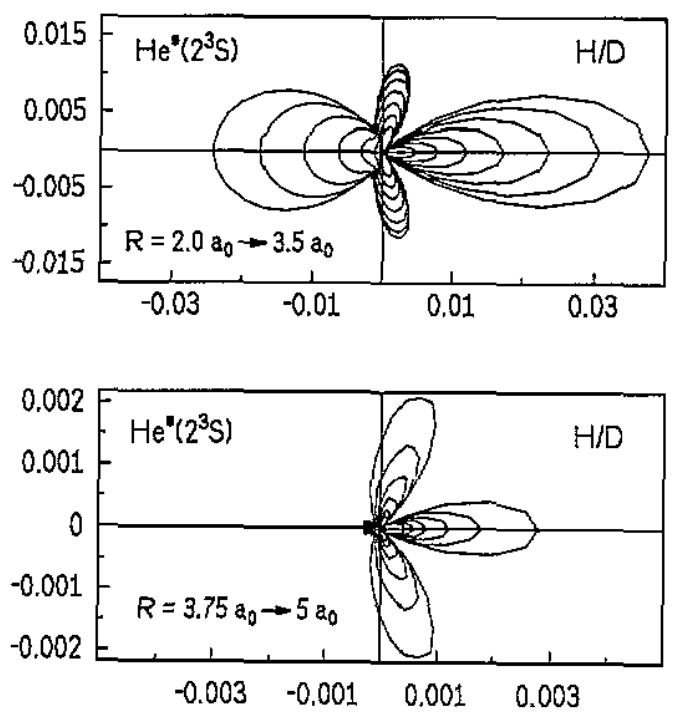

Figure 10. $A b$ initio results for the internal angular distribution $I_{\mathrm{AD}}(\vartheta, R)$ of electron emission from the collision complex $\left[\mathrm{He}\left(2^{3} \mathrm{~S}\right)-\mathrm{H} / \mathrm{D}\right]^{*}$ for short and intermediate internuclear distances $R$. The parameter $R$ is varied in steps of $\Delta R=0.25 a_{0}$, starting at $R=$ $2.0 a_{0}$ (outer curve of upper diagram) to $R=3.5 a_{0}$ (inner curve) and from $R=3.75 a_{0}$ to $R=5 a_{0}$ (lower diagram). The intensity scale of the polar plot is proportional to the autoionization width $\Gamma(R) / 2 \pi$ (au).

The correlation between the IAD and the electron energy spectrum becomes particularly clear at the characteristic steps in the AI regime at energies of $E_{\mathrm{el}} \simeq 7.55 \mathrm{eV}, 7.3 \mathrm{eV}$ and $7.1 \mathrm{eV}$, mentioned above. These onsets (see figure 8) are due to heavy particle partial wave contributions of small $J^{*}\left(0 \hbar \leqslant J^{*} \leqslant 5 \hbar\right)$ and their enhancement in forward and backward direction results from the combination of two effects: (i) for small $J^{*}$, the 
internuclear axes of the collision system at the moment of autoionization are oriented in a rather narrow angular range around the relative velocity direction, as shown by classical trajectory calculations (Merz et al 1992); (ii) at distances close to $R \approx 2.3 a_{0}$, where the electronic transitions to the AI channels occur, the $\mathrm{AD}$ shows strong forward and backward maxima (see figure 10 ). The angular variation of the quasi-associative ionization features can be explained by similar considerations. These peaks are connected with transitions to specific ro-vibrational $\mathrm{HeD}^{+}$states. Therefore it is also possible to correlate the orientation of the internuclear axis and the IAD (at $R \approx 2.4 a_{0}$ ) with the observed intensity variations (Merz et al 1992, 1993). The Airy pattern, however, is formed by contributions from all partial waves, and a broad range of internuclear distances and orientations is involved. The smooth change of the Airy oscillations when

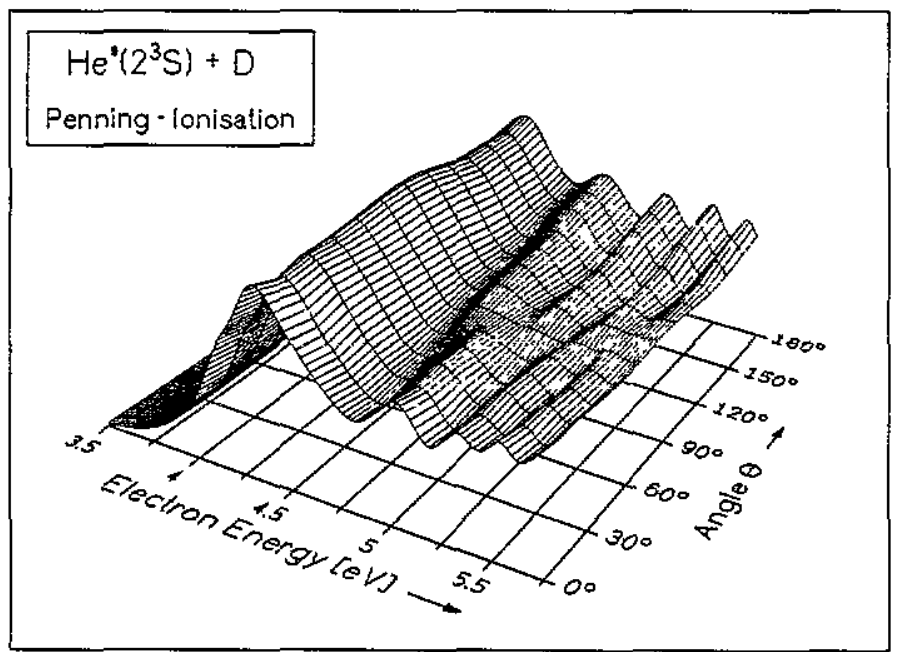

Figure 11. Theoretical angle-differential Penning ionization electron spectra for $\mathrm{He}^{*}\left(2^{3} \mathrm{~S}\right)+\mathrm{D}$ collisions, based on $a b$ initio potential data and partial autoionization amplitudes (figures 4,9 and 10). The spectra are averaged over the experimental collision energy distribution and have a common intensity scale proportional to the cross section.

going from forward to backward emission is illustrated in figure 11. This graph shows the theoretical spectra in steps of $\Theta=15^{\mathrm{c}}$. As explained above for $\mathrm{He}^{*}+\mathrm{H}$ these spectra were folded with an energy resolution of $36 \mathrm{meV}$ and weighted with the relevant experimental collision energy distribution. It becomes apparent how the secondary Airy oscillations get weaker with growing angle $\Theta$, disappearing more or less between $\Theta=60^{\circ}$ and $90^{\circ}$ and returning with a kind of 'phaseshift' in backward direction in good agreement with the experimental results of figure 8 . The main Airy peak changes only little in height and is almost fixed in position and width.

In figure 12 we directly compare the full theoretical and experimental electron spectra for $\mathrm{He}^{*}\left(2^{3} \mathrm{~S}\right)+\mathrm{D}$ at the three angles $\Theta=0^{\circ}, 90^{\circ}$ and $180^{\circ}$, respectively. This diagram confirms the satisfying agreement between theory and experiment: the changes in the fine-structured AI part are just as well reproduced as the positions and the angledependent modulation of the Airy oscillation. Comparison of the other spectra of figure 8 , not presented in figure 12 , shows the same good agreement. Some minor differencesparticularly regarding the shape of the secondary Airy peaks-can at least in part be traced to an incomplete treatment of the angular spread of the atomic deuterium beam, similar as for the hydrogen data in figure 6. 


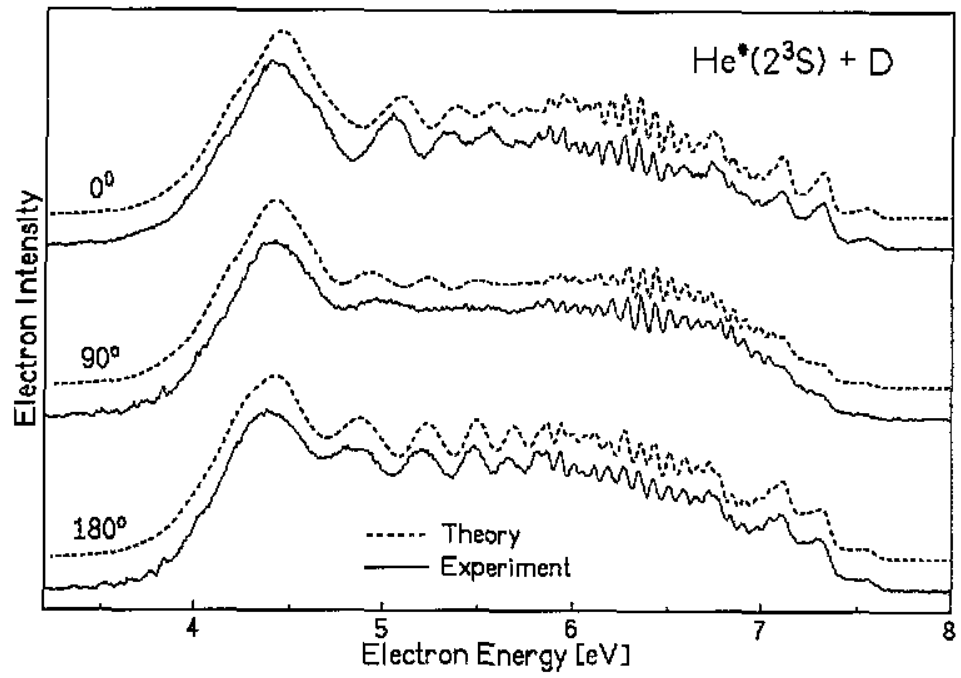

Figure 12. Comparison between experimental (full curve) and theoretical (broken curve) electron spectra for the detection angles $\Theta=0^{\circ}, 90^{\circ}$ and $180^{\circ}$. For clarity, the calculated spectra, which were averaged over the experimental collision energy distribution and folded with a resolution of $\Delta \varepsilon=36 \mathrm{meV}$, were displaced on the intensity scale.

\section{Summary and outlook}

We have presented experimental and theoretical work on the benchmark systems $\mathrm{He}^{*}\left(2^{3} \mathrm{~S}\right)+\mathrm{H} / \mathrm{D}\left(1^{2} \mathrm{~S}\right)$ for Penning and associative ionization processes. Our theoretical spectra, based on accurate $a b$ initio calculations for interaction potentials and partial autoionization amplitudes and a full treatment of the dynamics in local complex potential approximation, are found to be in good agreement with angle-dependent, highresolution experimental electron energy spectra of the systems $\mathrm{He}^{*}\left(2^{3} \mathrm{~S}\right)+\mathrm{H} / \mathrm{D}\left(1^{2} \mathrm{~S}\right)$. It turns out that the local complex potential method in the frame of the BornOppenheimer approximation is a valid approach for the theoretical description of these autoionizing collision complexes.

Recently, we have also investigated the autoionization dynamics of collisions between metastable helium atoms in the $2^{1} \mathrm{~S}$ state and atomic hydrogen/deuterium. The $a b$ initio results for the complex coupling elements and the IAD of electron emission from the quasi-molecules ( $\left.\mathrm{He}\left(2^{1} \mathrm{~S}\right) \mathrm{H} / \mathrm{D}\right)^{*}$ are quite different from those for the ${ }^{3} \mathrm{~S}$ system, shown in figures 9 and 10. A detailed comparison of angle-differential experimental spectra and theoretical spectra, again based on the local complex potential approximation, will be published elsewhere (Movre et al 1994).

Beyond that, we have extended our angle-dependent experimental studies to metastable neon atoms colliding with atomic hydrogen. The $\mathrm{Ne}^{*}\left({ }^{3} \mathrm{P}_{2}\right)+\mathrm{H}$ electron energy spectrum (Lorenzen et al 1983), which is very similar in shape and energy spread to the $\mathrm{He}^{*}\left(2^{3} \mathrm{~S}\right)+\mathrm{H}$ spectrum, also shows clear changes with electron detection angle. Interestingly, the angular variation of the AI structure in forward and backward direction is significantly different from that of the helium case. Details of the spectra and a possible model for the internal angular distribution of electron emission will be discussed in a forthcoming publication (Merz et al 1994).

In the near future we plan to investigate ionization in collisions of metastable argon and krypton atoms with hydrogen and deuterium atoms. In contrast to helium and 
neon, for these two species the excitation energy in the entrance channel lies asymptotically below the ionization limit of the exit channel. Therefore, the entrance channel potential interacts with the ionic continuum only at short internuclear distances. In a first step we want to clarify to what extent the two metastable states $\left({ }^{3} \mathrm{P}_{0},{ }^{3} \mathrm{P}_{2}\right)$ of argon and krypton actually lead to associative ionization. This will be done by measuring the total molecular ion yield $\left(\mathrm{ArH}^{+} / \mathrm{ArD}^{+}\right.$and $\left.\mathrm{KrH}^{+} / \mathrm{KrD}^{+}\right)$resulting from a crossed beam scattering arrangement between a state-selected metastable beam and a diffusive atomic hydrogen/deuterium beam. In a second step we intend to measure again the angular dependence of the electron energy spectrum.

A challenging task will be the theoretical description of these kinds of autoionizing collision complexes since the entrance and exit channel potential curves are not well separated as in the case of $\mathrm{He}^{*}$ and $\mathrm{Ne}^{*}+\mathrm{H}, \mathrm{D}$. Consequently, the conditions for the local complex potential approximation are not, or less well, obeyed. In particular, the local approach is invalid for electrons near threshold. It may be interesting to explore possible refinements of the local approximation and to find out whether a diabatic picture with static coupling or an adiabatic picture with dynamic coupling to the continuum is more appropriate (see also Niehaus 1981, Weiner et al 1990, Dulieu et al 1991).

\section{Acknowledgments}

This work has been supported by the Deutsche Forschungsgemeinschaft (SFB 91 and Ho 427/19-1) and through Graduiertenkolleg 'Laser- und Teilchenspektroskopie'. One of us (MM) acknowledges the hospitality of Fachbereich Chemie and Fachbereich Physik at the University of Kaiserslautern and financial support by the Deutsche Forschungsgemeinschaft (436 KRO-17).

\section{Appendix}

Photoelectron intensities (peak areas) for $\mathrm{He}(\mathrm{I} \alpha)+\mathrm{O}_{2}$ for electron detection at $\Theta=\$ 4.7^{\circ}$ (Bregel 1987) and corresponding values for $\Theta=90^{\circ}$ according to $I(\Theta)=$ $I_{0}\left[1+\beta / 2\left(\frac{3}{2} \sin ^{2}(\Theta)-1\right)\right]$ with $\beta$ taken from Kreile and Schweig (1980).

\begin{tabular}{lllrrr}
\hline Ionic state & & $E_{\mathrm{el}}(\mathrm{eV})$ & \multicolumn{1}{c}{$r\left(54.7^{\circ}\right)$} & \multicolumn{1}{l}{$\beta$} & \multicolumn{1}{l}{${ }\left(90^{\circ}\right)$} \\
\hline $\mathrm{O}_{2}^{+} \mathrm{X}^{2} \Pi_{\mathrm{v}}$ & 0 & 9.134 & 44.8 & -0.23 & 42.2 \\
& 1 & 8.900 & 100.0 & -0.28 & 93.0 \\
& 2 & 8.673 & 90.7 & -0.33 & 83.2 \\
& 3 & 8.450 & 46.1 & -0.39 & 41.6 \\
& 4 & 8.230 & 13.3 & -0.31 & 12.3 \\
$\mathrm{O}_{2}^{+} \mathrm{a}^{4} \Pi_{\mathrm{u}}$ & 0 & 5.116 & 3.4 & 0.45 & 3.8 \\
& 1 & 4.990 & 11.8 & 0.47 & 13.2 \\
& 2 & 4.868 & 22.7 & 0.45 & 25.3 \\
& 3 & 4.748 & 31.7 & 0.43 & 35.1 \\
& 4 & 4.629 & 36.9 & 0.44 & 41.0 \\
& 5 & 4.514 & 39.2 & 0.43 & 43.4 \\
$\mathrm{O}_{2}^{+} \mathrm{b}{ }^{4} \Sigma_{\mathrm{v}}$ & 0 & 3.046 & 88.6 & 0.52 & 100.1 \\
& 1 & 2.902 & 76.8 & 0.61 & 88.5 \\
& 2 & 2.763 & 51.3 & 0.63 & 59.4 \\
& 3 & 2.627 & 31.8 & 0.59 & 36.5 \\
& 4 & 2.496 & 17.4 & 0.62 & 20.18 \\
\hline
\end{tabular}


Appendix. (continued)

\begin{tabular}{llllll}
\hline lonic state & & $E_{\mathrm{el}}(\mathrm{eV})$ & $I\left(54.7^{\circ}\right)$ & $\beta$ & $I\left(90^{\circ}\right)$ \\
\hline $\mathrm{O}_{2}^{+} \mathrm{B}^{2} \Sigma_{\mathrm{B}}$ & 0 & 0.921 & 45.6 & 1.07 & 57.8 \\
& 1 & 0.783 & 55.0 & 1.07 & 69.7 \\
& 2 & 0.652 & 46.6 & 1.07 & 59.1 \\
& 3 & 0.524 & 34.5 & 1.02 & 43.3 \\
& 4 & 0.402 & 20.5 & 1.02 & 25.7 \\
\hline
\end{tabular}

\section{References}

Bardou F, Emile O, Courty J M, Westbrook C I and Aspect A 1992 Europhys, Lett. 20 681-6

Barnett C F (ed) 1990 Atomic Data For Fusion Vol I Collisions of $\mathrm{H}, \mathrm{H}_{2}$, He and Li Atoms and Ions with Atoms and Molecules (Oak Ridge. TN: Oak Ridge National Laboratory)

Bieniek R J 1978 Phys. Rev. A 18 392-413

Bieniek R J, Müller M W and Movre M 1990 J. Phys. B: At. Mol. Opt. Phys. 23 4521-38

Bregel T 1987 Dissertation Universität Kaiserslautern (unpublished)

Brunetti B G and Vecchiocattivi F 1993 Current Topics in Ion Chemistry and Physics (New York: Wiley)

Dulieu O, Giusti-Suzor A and Masnou-Seeuws F 1991 J. Phys. B: At. Mol. Opt. Phys. 24 4391-408

Dunning F B, Rundel R D and Stebbings R F 1975 Rev. Sci. Instrum. 46 697-701

Ebding $T$ and Niehaus A $1974 Z$. Phys. 270 43-50

Gerber G and Niehaus A 1976 J. Phys. B: At. Mol. Phys. 9 123-36

Gray L G, Keiffer R S, Ratliff J M, Dunning F B and Walters G K 1985 Phys. Rev. A 32 1348-5!

Hoffmann V and Morgner $H 1979$ J. Phys. B: At. Mol. Phys. 12 2857-74

Hotop H, Illenberger E, Morgner H and Niehaus A 1971 Chem. Phys. Lett. 10 493-7

Huber K P and Herzberg G 1979 Constants of diatomic molecules (Princeton, NJ: van Nostrand)

Julienne P S, Smith A M and Burnett K 1993 Adv. At. Mol. Opt. Phys. 30 141-98

Klyucharev A N 1993 Sov. Phys.-Usp. 36 486-512

Kolos W and Peek J M 1976 Chem. Phys. 12 381-6

Kreile J and Schweig A 1980 J. Electron Spectrosc. Relat. Phenom. 20 191-211

Lett P D, Jessen P S, Phillips W D, Rolston S L, Westbrook C I and Gould P L 1991 Phys. Rev. Lett. 67 $2139-42$

Lorenzen J, Morgner H, Bußert W, Ruf M-W and Hotop H 1983 Z. Phys. A 310 141-52

Magnier S, Millie Ph, Dulieu O and Masnou-Seeuws F $1993 \mathrm{~J}$. Chem. Phys. 98 7I13-25

Meijer H A J, Schohi S, Müller M W, Dengel H, Ruf M-W and Hotop H $1991 \mathrm{~J}$. Phys. B: At. Mol. Opt. Phys. 24 3621-32

Merz A 1993 Dissertation Verlag Shaker Aachen (ISBN 3-86111-561-1)

Merz A, Müller M W, Ruf M-W, Hotop H, Meyer W and Movre M 1989 Chem. Phys. Lett. 160 377-85

- 1990 Chem. Phys. 145 219-38

Merz A, Ruf M-W and Hotop H 1992 Phys. Rev. Lett. 69 3467-70

Merz A, Ruf M-W, Hotop H, Movre M and Meyer W 1993 The Physics of Electronic and Atomic Collisions, Proc. 18th ICPEAC (Aarhus, Denmark, 1993) (AIP Conf. Proc, 295) ed T Andersen, B. Fastrup, F Folkmann, $\mathrm{H}$ Knudsen and N Andersen (New York: AIP) pp 852-60

Merz A, Ruf M-W and Hotop H 1994 Z. Phys. D in press

Micha D A and Nakamura H 1975 Phys. Rev. A 11 1988-93

Miller W H $1970 \mathrm{~J}$. Chem. Phys. 52 3563-72

Mitsuke K, Kusafuka K and Ohno K 1989 J. Phys. Chem. 93 3062-8

Morgner H 1978 J. Phys. B. At. Mol. Phys. 11 269-80

Morgner $\mathrm{H}$ and Niehaus A 1979 J. Phys. B: At. Mol. Opt. Phys. 12 1805-20

Movre $M$ and Meyer W $1994 \mathrm{~J}$. Chem. Phys. submitted

Movre M, Meyer W, Merz A, Ruf M-W and Hotop H 1994 Chem. Phys. Lett. in press

Müller M W, Bussert W, Ruf M-W, Hotop H and Meyer W 1987 Phys. Rev. Lett. 59 2279-82

Müller M W, Merz A, Ruf M-W, Hotop H, Meyer W and Movre M 1991 Z. Phys. D 21 89-112

Nakamura H 1968 J. Phys. Soc. Japan 24 1353-65

- 1969 J. Phys. Soc. Japan 26 1473-79

Niehaus A 1981 Adv. Chem. Phys. 45 399-486 
Ruf M-W, Yencha A J and Hotop H 1987 Z, Phys. D 5 9-20

Siska P E 1993 Rev. Mod. Phys. $65337-412$

Tellinghuisen J 1985 Adv. Chem. Phys. $60299-369$

Urbain X 1993 The Physics of Electronic and Atomic Collisions, Proc. 18th ICPEAC (Aarhus, Denmark, 1993) (AIP Conf. Proc. 295) ed T Andersen, B. Fastrup, F Folkmann, H Knudsen and N Andersen (New York: AIP) pp 645-53

Yencha A J, Ruf M-W and Hotop H 1993 Z. Phys. D $27131-42$

Waibel H 1986 Dissertation Universităt Kaiserslautern (unpublished)

Waibel H, Ruf M-W and Hotop H 1988 Z. Phys. D 9 19!-207

Weiner J, Masnou-Seeuws F and Giusti-Suzor A 1990 Adv. At. Mol. Phys. 26 209-96 